\title{
INVESTIGAÇÃO DA ADESÃO MEDICAMENTOSA EM IDOSOS QUE FAZEM USO DE POLIFARMÁCIA: RELATO DE EXPERIÊNCIA
}

\author{
Maria Eduarda da Silva Rodrigues ${ }^{1}$, Girleide Santos do Nascimento ${ }^{1}$, Lucielly \\ Batista de Medeiros ${ }^{1}$, Mariana Albernaz Pinheiro de Carvalho ${ }^{2}$ \\ ${ }^{1}$ Curso de Bacharelado em Enfermagem, Unidade Acadêmica de Enfermagem, Universidade \\ Federal de Campina Grande, Cuité-PB, Brasil. \\ ${ }^{2}$ Prof ${ }^{a}$ Unidade Acadêmica de Enfermagem , Universidade Federal de Campina Grande, Cuité, \\ PB, Brasil. \\ Email para correspondência: mariaeduarda15cd@gmail.com
}

\begin{abstract}
Resumo
Com o aumento da expectativa de vida da população, o número de idosos vem aumentando e isso tem gerado aumento das doenças crônicas não transmissiveis e comorbidades, o que ocasiona o consumo de múltiplos fármacos. Objetivou-se relatar a experiência vivenciada por acadêmicas do curso de Bacharelado em Enfermagem durante a coleta de dados de pesquisa vinculada ao Programa de Iniciação Científica, envolvendo polifarmácia e adesão medicamentosa em idosos de um município da Paraíba. Relato de experiência desenvolvido em janeiro de 2021. Foram consultados os registros e anotações realizadas no caderno de campo das pesquisadoras, incluindo elementos que não foram contemplados nos instrumentos de coleta do estudo subsidiário. Durante a coleta, os idosos relataram abandono intencional no tratamento, abandono devido crenças religiosas, esquecimento dos horários de consumo dos medicamentos e falta de medicamentos no serviço de fármacia popular. Destaca-se ainda sentimentos de tristeza, solidão e abandono que influenciavam na adesão aos fármacos prescritos, como também a contribuição da família e cuidador nesse processo. Tal experiência trouxe contribuições relevantes para a vida acadêmica e pessoal das pesquisadoras, pois pôde-se observar, na prática, vunerabilidade e desafios enfrentados pela população idosa em estudo.
\end{abstract}

Palavras-chave: polimedicação, idosos, adesão à medicação, enfermagem.

\begin{abstract}
With the increase in the population's life expectancy, the number of elderly people has been increasing and this has generated an increase in non-communicable chronic diseases and comorbidities, which lead to the consumption of multiple drugs. The objective was to report the experience lived by academics of the Bachelor of Nursing course during the collection of research data linked to the Scientific Initiation Program, involving polypharmacy and medication adherence in the elderly in a municipality in Paraíba. Experience report developed in January
\end{abstract}


2021. The records and notes taken in the field notebook of the researchers were consulted, including elements that were not included in the collection instruments of the subsidiary study. During data collection, the elderly reported intentional abandonment of treatment, abandonment due to religious beliefs, forgetting the consumption times of medicines and lack of medicines in the popular pharmacy service. It is also highlighted feelings of sadness, loneliness and abandonment that influenced the adherence to prescribed drugs, as well as the contribution of the family and caregiver in this process. This experience brought relevant contributions to the academic and personal lives of the researchers, as it was possible to observe, in practice, the vulnerability and challenges faced by the elderly population under study.

Keywords: polymedication, elderly, medication adherence, nursing.

\section{Introdução}

Com o aumento da expectativa de vida, o número de idosos vem aumentando significativamente em todo o mundo. Segundo o Departamento de Assuntos Econômicos e Sociais das Nações Unidas, esse grupo populacional deve crescer cerca de 16\% até 2050, chegando a 1,5 bilhões de indivíduos. Pesquisa nacional evidencia esse aumento, demonstrando assim, a transição demográfica vivenciada (UNITED NATIONS, 2019; IBGE, 2018).

Com o advento da transição demográfica, observa-se uma mudança epidemiológica que tem favorecido o aumento da prevalência de Doenças Crônicas Não Transmissíveis (DCNT) (REIS; JESUS, 2017). Assim, é comum entre idosos a presença de múltiplas comorbidades, o que tem contribuído para que a população idosa seja uma das mais medicalizadas da sociedade, visto que, para o manejo destas patologias, a tecnologia em saúde mais utilizada são os medicamentos. Quando o tratamento envolve a associação de múltiplos fármacos, caracteriza um fenômeno conhecido como polifarmácia. Embora não exista um consenso na literatura, a definição mais aceita para essa condição é o uso de cinco ou mais medicamentos (GOMES et al., 2019).

Apesar do uso de múltiplos medicamentos ser importante para o alívio de sintomas e tratamento de doenças, essa condição apresenta outra face: a utilização de vários fármacos está relacionada ao aumento do risco de interação medicamentosa, eventos adversos, declínio funcional, aumento do número de internações hospitalares e até mesmo o óbito (HERMANN et al., 2021).

Em pesquisa desenvolvida por Gomes et al. (2019), identificou-se que $47,7 \%$ dos idosos polimedicados eram não aderentes à terapia 
medicamentosa. A adesão à terapia prescrita refere-se ao comportamento participativo e ativo, voluntário e colaborativo do paciente para atingir o efeito terapêutico. Sendo assim, essa prática é importante para garantir os resultados terapêuticos ideais. Ademais, cabe ressaltar que a não adesão desencadeia desfechos clínicos negativos (KIM et al., 2019).

Tendo em vista que a não adesão medicamentosa desencadeia uma série de consequências que poderiam ser evitadas, como o aumento da demanda em serviços de saúde; agravamento e prolongamento do curso de enfermidades, além da elevação de casos de morbidade e mortalidade. Dessa forma, torna-se necessária uma monitorização do tratamento a fim de evitar esses agravos (ZAFANI et al., 2020; YANG et al., 2016; ABREU et al., 2019).

Para mitigar as complicações desencadeadas pela não adesão ao tratamento, cabe à Atenção Básica realizar o acompanhamento e a avaliação envolvendo a participação da equipe multiprofissional, por meio da incorporação de tecnologias diversas no cuidado à saúde, juntamente com o uso de metodologias ativas baseadas na reflexão e no respeito à autonomia e à individualidade de cada idoso (CARNEIRO; AYRES, 2021).

Frente ao exposto, o presente artigo tem como objetivo relatar a experiência vivenciada por acadêmicas do curso de Bacharelado em Enfermagem durante a coleta de dados de pesquisa vinculada ao Programa de Iniciação Científica, envolvendo polifarmácia e adesão medicamentosa em idosos de um município da Paraíba.

\section{Metodologia}

Trata-se de um relato de experiência derivado da coleta de dados de pesquisa vinculada ao Programa Institucional de Bolsas de Iniciação Científica (PIBIC) da Universidade Federal de Campina Grande (UFCG), campus Cuité-PB, intitulada "Avaliação da relação entre polifarmácia e adesão medicamentosa em idosos". O estudo em questão buscou investigar a prevalência de polifarmácia e caracterizar a adesão medicamentosa em idosos expostos ao uso de cinco ou mais medicamentos.

Para tal, a coleta de dados ocorreu durante o mês janeiro de 2021, por meio de dois instrumentos: um questionário sobre o perfil sociodemográfico e uso de medicamentos para os idosos em uso de polifarmácia e o teste de 
Medida de Adesão ao Tratamento (MAT). A coleta foi realizada por três discentes do curso de bacharelado em Enfermagem da UFCG campus Cuité. A amostra foi composta de 231 idosos (com idade igual ou superior a 60 anos) residentes na zona urbana do município já referido anteriormente.

Tendo em vista que a coleta envolveu uma quantidade numerosa de idosos, identificou-se que apesar de serem utilizados instrumentos técnicos e objetivos de investigação, as pesquisadoras abordaram os participantes de maneira acolhedora e atenta aos relatos que eram evocados, registrando suas impressões e aspectos que não eram contemplados nos instrumentos de coleta do estudo subsidiário no caderno de campo. Assim, os participantes enxergaram naquele momento, uma oportunidade amistosa de diálogo e a utilizavam para conversar, partilhar experiências e amenizar sentimentos negativos, experiências essas que eram registradas no caderno de campo.

Cabe destacar que os participantes foram informados sobre os aspectos abordados na pesquisa, e em seguida, o Termo de Consentimento Livre Esclarecido (TCLE) fora apresentado e assinado em duas vias. A pesquisa que subsidiou a construção deste relato considerou a Resolução № 466/2012 do Conselho Nacional de Saúde e a realizou-se a coleta após aprovação do Comitê Ética em Pesquisa com parecer no 4.487 .645 (BRASIL, 2012).

\section{$3 \quad$ Resultados e discussão}

A coleta de dados é uma etapa crucial da pesquisa científica. Quando esse processo ocorre de forma direta com o participante, pode originar informações que por vezes não são registradas nos instrumentos ou em artigos.

Ao iniciar o diálogo sobre os medicamentos, alguns idosos relataram o abandono do tratamento de forma voluntaria, atitude que reforça que a não adesão à terapia medicamentosa também pode ocorrer de forma intencional, não somente devido a senescência ou outros fatores que caracterizam a não adesão sem intencionalidade (GOMES et al., 2019).

Oliboni e Castro (2018) afirmam que a não adesão intencional é a decisão ativa do paciente de não seguir com o regime terapêutico. Essa decisão pode estar relacionada às crenças, circunstâncias vivenciadas, prioridades ou preferências pessoais, além das experiências na motivação de uso do medicamento. Trazendo para este estudo, cabe destacar um episódio de 
abandono no consumo de medicamentos em razão de crenças religiosas por parte de uma idosa participante da pesquisa.

Relatos comuns entre os idosos foram o esquecimento no tocante ao horário correto para o consumo e os preços dos medicamentos. Confrontandose essa problemática com a realidade encontrada, Gomes et al. (2019) identificaram em seu estudo que $38,8 \%$ dos idosos apontaram o esquecimento como um fator relacionado à adesão ao tratamento farmacológico. Desse modo, o referido autor, identificou que o preço dos fármacos também interfere no uso correto de medicamentos, fator esse que tende a inviabilizar a aquisição dos mesmos por parte do usuário idoso e consequentemente o seu uso. Tal condição foi constatada durante a coleta de dados desta pesquisa, visto que, em determinadas circunstâncias, os fármacos não estavam disponíveis na farmácia popular, o que comprometia a sua utilização por parte dos idosos, pois alguns não possuíam condições financeiras para comprá-lo.

Durante o desenvolvimento da coleta, foi possível observar ainda que alguns idosos relataram a influência de morarem sozinhos, além da perda do cônjuge ou familiar como fator relacionado a não adesão medicamentosa. Nesse sentido, destaca-se o caso de uma das entrevistadas, ao relatar que a solidão e o sentimento de tristeza após o falecimento do cônjuge eram muitas vezes os fatores desencadeantes de displicência com o horário de uso do medicamento, haja vista que essa prática remetia às lembranças de momentos vivenciados com o companheiro, o que causava tristeza e saudade.

Ainda em se tratando de relatos de solidão, abandono e tristeza, visualizou-se que tais sentimentos afetavam diretamente a aderência medicamentosa. Portanto, vale ressaltar a importância e a presença da família, cuidador ou apoio de terceiros, como potenciais facilitadores do uso correto de medicamentos, a exemplo do caso de uma participante que mesmo sendo viúva, contava com o suporte da filha no acompanhamento direto de suas atividades diárias, incluindo a terapia medicamentosa, uma vez que a idosa fazia o uso diário de 12 fármacos e após avaliação da adesão ao tratamento por meio do instrumento MAT, revelou ser uma das pessoas que aderiu ao tratamento proposto para suas comorbidades.

A idosa ainda enfatizou o quanto a presença da filha era importante, reforçando sua participação não apenas na prática de consumo 
medicamentoso, mas na oferta de amparo e cuidado, o que reforça a necessidade de garantia de bem-estar, dignidade e direito à vida, assim como dispõe a Constituição Federal em seu artigo número 230 (BRASIL, 1988).

Além disso, pôde-se verificar que a utilização de diversas estratégias como: armazenar os comprimidos consumidos ao longo do dia em locais diferentes e memorizar o formato da embalagem ou a cor do medicamento, sobretudo quando se tratam de idosos analfabetos, facilitava à adesão. Tais alternativas também foram evidenciadas em estudo realizado por Silva e Spinillo (2016).

Desse modo, embora existam dificuldades na adesão ao tratamento por diversos fatores já mencionados, pôde-se observar que o uso de estratégias diversas possibilita o consumo de medicamentos de maneira segura, minimizando os impactos gerados devido à complexidade da terapêutica. Sendo assim, pesquisadores inferem que fica evidente a necessidade da implementação de ações que promovam o uso racional de medicamentos e contribuam para uma melhor compreensão dos idosos acerca da temática, além de estimular a qualificação de profissionais e cuidadores envolvidos na assistência a usuários expostos a tais condições (CORRALO et al., 2018).

\section{Conclusão}

A experiência proporcionada por este estudo permitiu reforçar a compreensão de que a população idosa está cada vez mais exposta a comorbidades e como medida de tratamento, tem-se a necessidade do consumo de vários fármacos. Além disso, o estudo ressalta que os idosos usuários de múltiplos medicamentos concomitantes, apresentam dificuldades referentes à posologia, condições financeiras e outros fatores que influenciam na adesão correta ao tratamento. Foi possível visualizar que os idosos que participaram da pesquisa relataram fazer uso de diversas estratégias facilitadoras desse consumo.

Dessa forma, essa experiência contribuiu para o desenvolvimento de um olhar mais sensível e crítico das pesquisadoras, futuras profissionais de enfermagem sobre a problemática. Permitiu ainda produzir reflexões sobre alguns fatores relacionados à não adesão e a importância de se considerar cada idoso em sua singularidade, sobretudo quando se tratam de demandas 
psicológicas e emocionais que podem influenciar o padrão de consumo medicamentoso.

Assim, destaca-se a necessidade do acompanhamento de idosos em uso de múltiplos medicamentos pelos profissionais de saúde atuantes especialmente na Atenção Básica, além da urgência em se promover ações educativas que conscientizem essa polulação acerca do consumo correto, de forma a se obter resultados desejáveis a partir do tratamento proposto.

\section{Referências}

ABREU, D. P. G. et al. Fatores Comportamentais Associados à Adesão Medicamentosa Em Idosos Em Atendimento Ambulatorial. Revista de Enfermagem do Centro Oeste Mineiro. v. 9, 2019. Disponível: <http://seer.ufsj.edu.br/index.php/recom/article/view/3025>. Acesso em: 07 jun. 2021.

BRASIL. Constituição (1988). Constituição da República Federativa do Brasil. Brasília, DF: Senado Federal: Centro Gráfico, 1988.

BRASIL. Ministério da Saúde. Conselho Nacional de Saúde. Resolução no 466, de 12 de dezembro de 2012. Diário Oficial [da] República Federativa do Brasil.

CARNEIRO, J. L. et al. Saúde do idoso e atenção primária: autonomia, vulnerabilidades e os desafios do cuidado. Revista de Saúde Pública, v. 55, p. 29, 2021. Disponível em: <https://www.scielo.br/j/rsp/a/hGd5rfTFM3BWBQNnrbLvTZS/abstract/?lang=pt>. Acesso em: 07 jun.2021

CORRALO, V. S. et al . Polifarmácia e fatores associados em idosos diabéticos. Rev. salud pública, Bogotá, v. 20, n. 3, p. 366-372, jun. 2018. Disponível em $<\mathrm{http}$ ://www.scielo.org.co/scielo.php?script=sci_arttext\&pid=S01240064201800030036 $6 \& l n g=p t \& n r m=i s o>$. Acesso em: 08 jun. 2021.

GOMES, D. et al. Daily Medication Management and Adherence in the Polymedicated Elderly: A Cross-Sectional Study in Portugal. Int J Environ Res. Public. Health. v. 17, n. 1, 2019. Disponível em: <https://www.ncbi.nlm.nih.gov/pmc/articles/PMC6981635/>. Acesso em: 27 maio 2021.

HERMANN M. et al. Polypharmacy and Potential Drug-Drug Interactions in HomeDwelling Older People - A Cross-Sectional Study. J Multidiscip Healthc. v. 14, p. 589597, 2021. Disponível: <https://pubmed.ncbi.nlm.nih.gov/33727821/>. Acesso em:29 maio 2021.

IBGE, Diretoria de Pesquisas, Coordenação de Trabalho e Rendimento, Pesquisa Nacional por Amostra de Domicílios Contínua 2018. Disponível em $<$ https://biblioteca.ibge.gov.br/visualizacao/livros/liv101654_informativo.pdf.> Data de acesso: 27 maio 2021.

$\mathrm{KIM}$, S. J. et al. Impact of number of medications and age on adherence to antihypertensive medications: A nationwide population-based study. Medicine, v. $98 \mathrm{n}$. 
49, 2019. Disponível em: <https://www.ncbi.nlm.nih.gov/pmc/articles/PMC6919523/>. Acesso em: 29 maio 2021.

OLIBONI, L. S., CASTRO, M. S. Adesão à farmacoterapia, que universo é esse? Uma revisão narrativa. Clin Biomed Res. v. 38, n. 2, 2018. Disponível em: <https://seer.ufrgs.br/index.php/hcpa/article/view/80552>. Acesso em: 29 maio 2021.

REIS, K. M. C., JESUS, C. A. C. Relação da polifarmácia e polipatologia com a queda de idosos institucionalizados. Texto contexto - enferm., Florianópolis, v. 26, n. 2, 2017. Disponível: <https://www.scielo.br/j/tce/a/pxpbpth8WyWPkTtWcX9Y5ML/?lang=pt\&format=pdf>. Acesso em: 27 maio 2021.

SILVA, C. H., SPINILLO, C. G. Dificuldades e estratégias no uso de múltiplos medicamentos por idosos no contexto do design da informação. Estudos em design, v. 24, n. 3, 2016.

UNITED NATIONS. World Population Ageing 2019. Department of Economic and Social Affairs. New York, 2020.

YANG, Z. et al. Association of antihypertensive medication adherence with healthcare use and medicaid expenditures for acute cardiovascular events. Med Care; v. 54, n. 5, p.504-11., 2016.

ZAFANI, G. C. et al. A relação da polifarmácia e adesão medicamentosa com compensação ou não de doenças em idosos residentes com familiares, cônjuges ou sozinhos. Archives Of Health Investigation, v. 9, n. 5, p. 410-413, 2020. Disponível em: <https://www.archhealthinvestigation.com.br/ArcHI/article/view/4846>. Acesso em: 07 jun. 2021. 